\title{
Reconfiguração de Redes de Distribuição de energia elétrica utilizando unineuron e nullneuron
}

\author{
Mariane M. Santana* Pyramo P. da Costa ${ }^{* *}$ \\ Maury M. Gouvêa Jr ${ }^{* * *}$ Fabrício P. Lucas ${ }^{* * * *}$ \\ * Faculdade de Engenharia de Controle $\mathscr{G}$ Automação, Pontifícia \\ Universidade Católica de Minas Gerais, MG, (e-mail: \\ marianemilitaosantana@gmail.com). \\ ** Faculdade de Engenharia Elétrica, Pontifícia Universidade Católica \\ de Minas Gerais, MG, (e-mail: pyramo@pucminas.br). \\ *** Faculdade de Engenharia Elétrica, Pontifícia Universidade Católica \\ de Minas Gerais, MG, (e-mail: maury@pucminas.br) \\ **** Faculdade de Engenharia de Controle \& Automação, Pontifícia \\ Universidade Católica de Minas Gerais, MG, (e-mail: \\ fpl1992@yahoo.com.br).
}

\begin{abstract}
The reconfiguration of electric power distribution networks is an indispensable tool in the planning and operation of the distribution system. The presented work describes a multi-objective methodology for distribution networks reconfiguration, by using unineurons and nullneurons. The new configuration should minimize the total active power loss and minimize feeder loading without violating operational constraints. Tests with the algorithms were performed with IEEE 33 bus electrical system. The obtained results were compared with those from the literature, demonstrating that the developed methodology is promising and provides good results, taking into account all the physical and operational constraints of the problem.

Resumo: A reconfiguração de redes de distribuição de energia elétrica é uma ferramenta indispensável no planejamento e operação do sistema de distribuição. O presente trabalho descreve uma metodologia multi-objetivo para reconfiguração de redes de distribuição, utilizando unineurons e nullneurons. A nova configuração deve minimizar a perda total de energia ativa e minimizar o carregamento do alimentador sem violar as restrições operacionais do sistema de distribuição. Os testes com os algoritmos foram realizados com o sistema elétrico IEEE 33 barras. Os resultados obtidos foram comparados com os da literatura, demonstrando que a metodologia desenvolvida é promissora e fornece bons resultados, levando em consideração todas as restrições físicas e operacionais do problema.
\end{abstract}

Keywords: Load Reconfiguration; Distribution System; Artificial neural networks; Nebulous Systems; Heuristic Methods.

Palavras-chaves: Reconfiguração de carga; Rede de Distribuição; Rede Neural Artificial; Sistemas Nebulosos; Métodos Heurísticos.

\section{INTRODUÇÃO}

O sistema de distribuição de energia elétrica é um sistema complexo, cuja condição de operação pode não permanecer constante. As várias contingências como, grandes variações de carga, falta de componentes (linhas de transmissão, transformadores, geradores, etc.) são mais comuns (Romano et al., 2013). Qualquer uma dessas condições faz com que parâmetros do sistema excedam os limites de segurança, resultando em um sistema inseguro. As técnicas mais praticadas para alívio de sobrecarga são o reescalonamento de geradores e/ou a reconfiguração das cargas.

\footnotetext{
* Agradecimento à FAPEMIG pelo suporte financeiro para desenvolvimento do presente trabalho.
}

As redes do sistema de distribuição de energia elétrica são operadas em uma configuração radial. A presença de diversas interconexões, entre diferentes alimentadores na mesma rede de distribuição permite configurações diversificadas para fornecer energia às cargas. Sendo assim, a reconfiguração da rede de distribuição pode ser utilizada com o objetivo de minimizar as perdas elétricas, melhorar perfil de tensão e balancear a carga entre os alimentadores (Li et al., 2007).

Diversos trabalhos sobre reconfiguração de redes elétricas estão presentes na literatura desde 1970. A minimização das perdas de potência ativa é a principal função objetivo utilizada. Dentre as técnicas largamente aplicadas na solução do problema de reconfiguração de redes elé- 
tricas de distribuição se destacam as técnicas heurísticas e meta heurísticas. Técnicas heurísticas nem sempre possuem como alvo uma solução ótima do problema. Possuem como ponto de partida uma solução viável. Baseiam-se em sucessivas aproximações direcionadas a um ponto ótimo. Logo, esses métodos costumam encontrar as melhores soluções possíveis para problemas, e não soluções exatas (Oliveira, 2016). Já as técnicas metaheurísticas constituemse da combinação de métodos heurísticos, aplicados em problemas gerais de alta complexidade e elevado espaço de solução. São geralmente aplicadas para buscas locais, porém suas características de busca inteligente, em muitos casos, permitem explorar novas regiões do espaço, podendo alcançar ótimos globais (Macedo, 2012).

Entre os métodos Heurístico e Metaheurísticos mais aplicados a problemas de otimização para reconfiguração de redes de distribuição, destacam-se, Branch and Bound (Merlin and Back, 1975; Romano et al., 2013) e Branch Exchange (Civanlar et al., 1988; Baran and Wu, 1989; Peponis and Papadopoulos, 1997; Bernardon et al., 2009; Junior, 2014) como métodos Heurísticos e PSO (Particle Swarm Optmization) (Tofis et al., 2017; Macedo, 2012; Atteya et al., 2017), Inteligência Artificial (Pandiarajan and Babulal, 2011; Bernardon et al., 2009; Berredo et al., 2011; Neto and Vellasco, 2016) e Colônia de formigas (Amin Heidari, 2017; Silva, 2010; Ghorbani et al., 2008) como métodos Metaheuríscos.

Um dos primeiros trabalhos relativo ao problema de reconfiguração de sistemas de distribuição de energia elétrica abordou a redução de perdas ôhmicas e foi proposto por Merlin and Back (1975). Neste trabalho é apresentado o método heurístico baseado na técnica de programação matemática inteira Branch and Bound.

Outra técnica heurística bastante utilizada é a Branch Exchange (Civanlar et al., 1988; Baran and Wu, 1989; Peponis and Papadopoulos, 1997; Bernardon et al., 2009; Junior, 2014). O método consiste em assumir inicialmente uma configuração radial e realizar a partir de então, vários procedimentos de comutação das chaves da rede na tentativa de produzir configurações novas e promissoras, sempre observando a radialidade da rede.O método se difere do Branch and Bound por garantir a radialidade do sistema e por estabelecer limites $\min / \max$ de tensão.

Os métodos metaheurísticos são também bastante utilizados, apesar de apresentarem elevado esforço computacional. Dentre eles, estão os métodos ACO (Ant Colony optimisation) por otimização de colônia de formigas e por enxame de partículas ou PSO (Particle Swarm Otimization). Ambos são exemplos de algoritmos evolucionários baseados no conceito de inteligência computacional.

O PSO é uma técnica inspirada na simulação de um sistema social. Ele simula a migração e agregação de um bando de aves procurando por comida e/ou local seguro. O método foi aplicado por (Tofis et al., 2017; Macedo, 2012; Ghorbani et al., 2008) para reconfiguração de uma rede de distribuição de energia elétrica e apresentou a desvantagem de considerar apenas uma função objetivo. Atteya et al. (2017) propôs também uma modificação do PSO (MPSO), onde a diferença entre ele e o o típico PSO está no espaço de busca seletiva aleatório filtrado para posição inicial, que é proposto para acelerar o algoritmo para alcançar a solução ótima.

Silva (2010), utiliza um novo algoritmo baseado na estrutura do método de otimização por colônia de formigas (ACO) para reconfiguração do sistema de distribuição. No método ACO, um conjunto de formigas artificiais (agentes) cooperam entre si a fim de encontrar soluções "ótimas"para problemas de otimização discreto e complexos. O algoritmo não garante radialidade do sistema, limitando assim o espaço de soluções à somente o conjunto de todas as topologias radiais conexas (Silva, 2010).Amin Heidari (2017), utiliza os métodos ACO e PSO em conjunto para a solução do problema de reconfiguração de sistemas de distribuição. Os resultados simulados mostraram que o algoritmo é efetivo na solução do problema de reconfiguração.

Outros autores utilizam de métodos de Inteligênia Artificial para solução do problema de reconfiguração de redes de distribuição (Pandiarajan and Babulal, 2011; Bernardon et al., 2009; Berredo et al., 2011; Neto and Vellasco, 2016). Pandiarajan and Babulal (2011)] utilizou a lógica Fuzzy para realizar remanejamento de carga e aliviar a sobrecarga no sistema de energia, porém este método não apresentou capacidade de minimizar a quantidade de carga a ser transferida. Neto and Vellasco (2016) utiliza Lógica Fuzzy em conjunto com Busca Tabu para solução do problema, porém a metodologia proposta não descartou a possibilidade de uma solução ruim. Já Berredo et al. (2011) e Neto and Vellasco (2016) apresentam resultados de pesquisas sobre o uso da abordagem de Bellman Zadeh para a tomada de decisão em um ambiente nebuloso para resolver o problema de reconfiguração. A abordagem de Bellman Zadeh permite a realização de um método computacionalmente efetivo, bem como rigoroso (do ponto de vista de obtenção de soluções) de análise de modelos multicritério. Seu uso permite preservar uma medida natural de incerteza na tomada de decisões e considerar índices, critérios e restrições de caráter qualitativo.

O objetivo deste trabalho é apresentar uma abordagem para obtenção de novas configurações radiais para o sistema de distribuição de Energia Elétrica. As novas configurações devem minimizar as perdas de potência ativa totais, e minimizar o carregamento dos alimentadores, sem violar as restrições operacionais.

Este artigo está organizado como segue. A seção 2 apresenta a formulação matemática utilizada para realizar o remanejamento de carga. A seção 3 apresenta a metodologia utilizada na pesquisa. A seção 4 apresenta os resultados obtidos com a pesquisa e a seção 5 apresenta a conclusão final do artigo.

\section{FORMULAÇÃO MATEMÁTICA DO PROBLEMA}

A partir da possibilidade de alteração das configurações da rede de distribuição, surge o problema de otimização conhecido como problema de reconfiguração de rede. Seu objetivo principal consiste em encontrar uma estratégia de operação ideal. Uma solução eficiente para esse problema requer a seleção da topologia de operação mais apropriada entre todas as configurações disponíveis. Examinar todas as alternativas de configuração torna o processo inviável para sistemas de distribuição reais, uma vez que o número 
de configurações viáveis cresce exponencialmente com o número de barras do sistema. Esse fato é conhecido como explosão combinatória (Bernardon et al., 2009).

Sendo assim, é proposto neste trabalho uma técnica Heurística (Branch Exchange) em conjunto com um método eficiente de tomada de decisão baseado em neurônios nebulosos Fuzzy. Neurônios nebulosos foi abordagem escolhida por apresentar algumas vantagens em relação a abordagens alternativas, devido ao paralelismo inerente das redes neurais. O objetivo é reduzir a perda de potência ativa total e minimizar o carregamento dos alimentadores, garantindo a radialidade do sistema e mantendo níveis de corrente e tensão adequados, no sentido de atender as exigências da agência reguladora.

\subsection{Funções objetivo e restrições}

A escolha das funções objetivo é uma etapa importante na modelagem do problema. Essa escolha deve refletir as necessidades do operador e auxiliar a tomada de decisão (Romano et al., 2013). Para o caso desenvolvido neste trabalho, foi considerado como função objetivo a minimização das perdas de potência ativa da rede elétrica e minimização do carregamento dos alimentadores em um problema de reconfiguração multiobjetivo.

Para minimização da perda de potência ativa do sistema de distribuição de energia elétrica, adotou-se como função objetivo a equação (1).

$$
\min f_{1}(x)=\sum_{j=1}^{N_{r}} R_{j} I_{j}^{2}
$$

Para minimização do carregamento dos alimentadores, adotou-se como função objetivo a equação (2).

$$
\min f_{2}(x)=\sum_{j=1}^{N_{r}} I_{j}
$$

Onde $I_{j}$ é a magnitude da corrente no ramo $j, R_{j}$ a resistência do ramo $j$ e $N_{r}$ o conjunto de ramos do sistema.Para que as configurações possam ser consideradas como viáveis, esta deve atender a algumas restrições, como:

(1) Radialidade;

O sistema é considerado radial quando a igualdade da equação (3) é satisfeita.

$$
n_{\text {linhas }}=n_{\text {barras }}-1
$$

Sendo, $n_{\text {linhas }}$ o número de linha (chaves) do sistema radial e $n_{\text {barras }}$ o número de barras do sistema.

(2) A magnitude da corrente de cada elemento deve estar dentro de um limite permitido;

$$
\left|I_{j}\right| \leq I_{\text {jmax }}
$$

Sendo $I_{j \max }$ o limite máximo da corrente no ramo $j$

(3) A magnitude da tensão de cada nó deve estar dentro de um intervalo permitido;

$$
V_{j \min } \leq V_{j} \leq V_{\text {jmax }}
$$

De acordo com a Agência Nacional de Energia Elétrica Brasileira (ANEEL), os limites de tensão nas barras da rede elétrica são divididos em três faixas, como apresentado na Tabela 1 . Onde $V_{j}$ é a tensão na $j_{t h}$ barra em p.u (Junior, 2014).
Tabela 1. Limites de Tensão

\begin{tabular}{|l|c|}
\hline \multicolumn{2}{|c|}{ Faixas de variação de Tensão } \\
\hline Adequada & $0,91 \leq V_{j} \leq 1,04$ \\
\hline Precária & $0,86 \leq V_{j}<0,91$ ou $1,04<V_{j} \leq 1,06$ \\
\hline Crítica & $V_{j}<0,86$ ou $V_{j}>1,06$ \\
\hline
\end{tabular}

\subsection{Cálculo do fluxo de Potência}

O fluxo de potência (FP) consiste de uma ferramenta básica para análise de sistemas elétricos de potência (SEP). Dado um sistema elétrico composto por subestação, alimentadores e barra de carga, utilizando o cálculo do fluxo de potência, é possível determinar valores de potência ativa e reativa, fasores de corrente e tensão, fator de potência e tensão elétrica em cada barra de carga (Chen et al., 2016; Yan et al., 2017; Macedo, 2012).

Para realizar o cálculo de fluxo de potência foi escolhido o pacote MATPOWER no MATLAB (Zimmerman et al., 2011), que utiliza o método Newton Raphson, que consiste na expansão da série de Taylor para múltiplas variáveis.

\subsection{Procedimento proposto para seleção das configurações}

Para seleção das configurações foi escolhido um procedimento de busca heurística, a qual é baseada na estratégia Branch Exchange. Portanto, uma configuração radial é assumida inicialmente e vários procedimentos de comutação são seguidos na tentativa de produzir configurações novas e promissoras, sempre observando a radialidade da rede. Estes procedimentos correspondem a um interruptor aberto e um interruptor fechado. Todas as configurações escolhidas pelo procedimento são classificadas de acordo com cada função objetivo definida e em relação as restrições estabelecidas, lembrando que soluções inviáveis são descartadas (Bernardon et al., 2009).

O fluxograma utilizado para selecionar a configuração candidata é mostrado na Figura 1. Onde, chave NA são as chaves normalmente abertas do sistema e chave NF são as chaves normalmente fechadas do sistema.

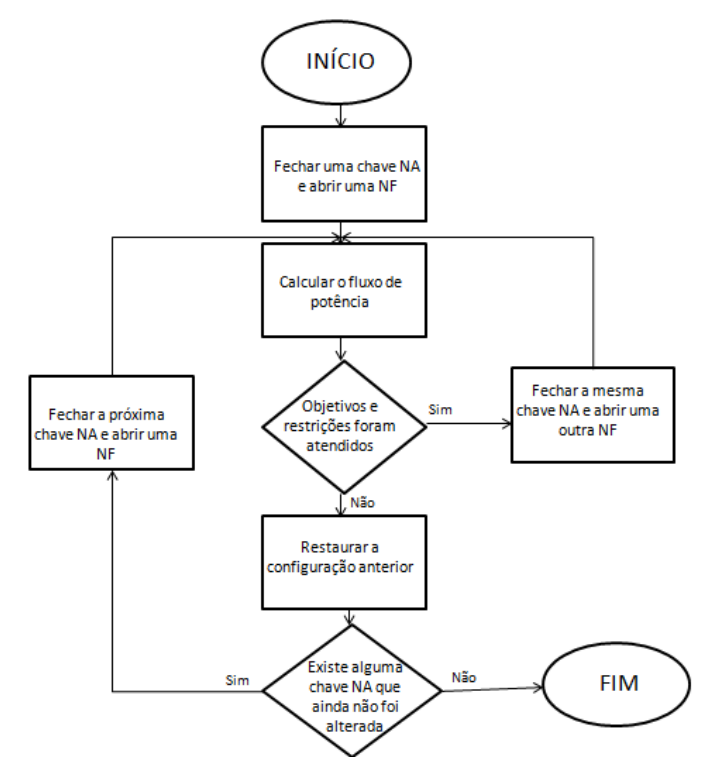

Figura 1. Seleção das configurações 


\subsection{Método de Bellman Zadeh}

Após definir quais as melhores configurações considerando cada função objetivo separadamente, é necessário definir quais configurações detêm os melhores resultados, uma vez que duas funções objetivo estão sendo consideradas. Por exemplo, quando uma configuração possui os melhores valores para perda de energia, mas existe uma outra melhor avaliada em relação ao carregamento dos alimentadores, é necessário decidir qual delas possui a melhor configuração geral, sendo assim, foi utilizado um algoritmo de tomada de decisão multicritério.

Em problemas de tomada de decisões multiobjetivo, diferentes funções objetivo podem ter diferentes naturezas e, consequentemente, são expressas em diferentes unidades e suas escalas não são comparáveis. Levando isso em consideração, a comparação da qualidade das soluções para cada função objetivo deve ser realizada em uma mesma escala (Junior, 2014). A equação (6) mostra a técnica utilizada para a transformação da função objetivo utilizadas neste trabalho. O resultado fornece a pertinência da função ao objetivo que será utilizado como entrada dos neurônios.

$$
F_{p_{2}}=\frac{\min _{p}(x)}{f_{p}(x)}
$$

A equação é utilizada para minimização da função objetivo. Onde, $F_{p}$ é o valor da função de pertinência, $f_{p}(x)$ é o valor da função objetivo $\max f_{p}$ é o valor máximo das funções objetivo e $\operatorname{minf}_{p}$ é o valor mínimo das funções objetivo.

O método de Bellman and Zadeh (1970) foi adotado como a primeira técnica de tomada de decisão neste trabalho, por apresentar eficiência no tratamento de critérios quantitativos e qualitativos na resolução de problemas. Quando é utilizada a abordagem Bellman Zadeh, toda função objetivo $f_{p}(x)$ é substituída por uma função objetivo nebulosa ou um conjunto nebuloso, como mostrado na equação (7).

$$
F_{p}=\left\{X, \mu_{f_{p}(x)}, p=1, \ldots, q\right\}
$$

Onde $\mu_{F_{p}(x)}$ é uma função de pertinência de $F_{p}$.

Assim a solução nebulosa $D$ é formada com a função de pertinência:

$$
\mu_{D}(x)=\min _{p=1, \ldots, q} \mu_{F_{p}}(x)
$$

Sua utilização permite obter uma solução que prove o grau máximo de pertinência à solução nebulosa $D$.

$$
\max \mu_{D}(x)=\operatorname{maxmin} \mu_{F_{p}}(x)
$$

Desta forma, a melhor configuração segundo o método de Bellman Zadeh é assumida quando ocorre a máxima intercessão entre as funções objetivo. Assim, do ponto de vista formal, o problema é multiobjetivo e substituído pelo problema max min, como mostra a equação (10)

$$
X^{0}=\operatorname{argmaxmin} \mu_{F_{p}}(x), p=1, \cdots, q
$$

No entanto, a busca por soluções baseadas na construção e solução de problemas maxmin representa uma visão pessimista em relação à avaliação da satisfação dos níveis de critérios, o que não é adequado em certas situações de tomada de decisão. Em particular, soluções em que um único objetivo tem um baixo nível de satisfação e outros critérios têm altos níveis de satisfação são considerados soluções ruins (Junior, 2014).

Levando isso em consideração, a análise multicritério foi complementada utilizando unineurons e nullneurons.

\subsection{Unineurons e Nullneurons}

Unineurons e nullneurons são neurônios nebulosos baseado em neurônios lógicos AND e OR, propostos por Pedrycz and Rocha (1993). São neurônios que podem variar entre um neurônio lógico do tipo AND até um neurônio lógico do tipo OR de forma a melhor se adaptar às necessidades do problema a ser resolvido (Hell, 2008). Além disso, a abordagem proposta permite o uso de diversas formas de representação das informações fornecidas por um decisor. Este decisor identifica a importância de cada função objetivo, utilizando pesos diferenciados para cada objetivo (Junior, 2014).

Como o próprio nome já diz, os unineurons são baseados no conceito de uninorma enquanto os nullneurons no conceito de nullnorma.

Uma uninorma e uma nullnorma é um operador fuzzy que generaliza a T-norma e a S-norma. Tanto as T-normas quanto as S-normas satisfazem as seguintes propriedades:

- Comutatividade: $a \cup b=b \cup a$;

- Associatividade: $a \cup(b \cup c)=(a \cup b) \cup c$;

- Monotocidade: se $b \leq c$ então $a \cup b \leq a \cup c$;

\subsection{Uninorma}

Uma uninorma $U$ é um operador binário com um elemento neutro $g \epsilon[0,1]$. O elemento neutro $g$ é claramente único e pode ser qualquer número de um intervalo unitário. Uma uninorma difere das T-normas e S-normas apenas no que diz respeito ao elemento neutro. Para T-normas e S-normas, tem-se valores fixos para os elementos neutros, enquanto, para as uninormas, este valor pode ser escolhido no intervalo $[0,1]$.

Desta forma, a característica de uma uninorma pode variar suavemente de um and puro para um or puro quando o elemento identidade varia de 1 para 0 . Quando $g=1$ leva a uma T-norma, quando $g=0$ leva a uma S-norma Yager and Rybalov (1996). Na literatura, é possível encontrar uma grande variedade de realizações de uninormas e nullnormas Yager and Rybalov (1996); Yager (2001). Uma das realizações mais usadas está vinculada à família de construtores mostrada na equação (11).

$$
U(x, y ; g)= \begin{cases}g T\left(\frac{x}{g}, \frac{y}{g}\right) & \text { se } x, y \epsilon[0, g] \\ g+(1-g) S\left(\frac{x-g}{1-g}, \frac{y-g}{1-g}\right) & \text { se } x, y \epsilon[g, 1] \\ \min (x, y) \text { oumax }(x, y) & \text { caso contrario }\end{cases}
$$

Onde $\mathrm{T}$ é uma T-norma, S uma S-norma, $x \epsilon[0,1]^{n} \mathrm{e}$ $y \epsilon[0,1]^{n}$. Neste trabalho, a T-norma foi considerada como operador min, e a S-norma como operador max. 


\subsection{Nullnorma}

Uma nullnorma é um operador binário com um elemento de absorção $u \epsilon[0,1]$. O elemento de absorção $u$ é claramente único. A nullnorma também é uma generalização de T-normas e S-normas, pois, por denição, quando $u=1$ leva a uma S-norma, quando $u=0$ leva a uma T-norma (Yager and Rybalov, 1996).

Uma realização específica de nullnorma é apresentada e pode ser traduzida na família de construtores mostrada na equação (12).

$$
N(x, y ; u)= \begin{cases}u S\left(\frac{x}{u}, \frac{y}{u}\right) & \text { se } x, y \epsilon[0, u] \\ u+(1-u) T\left(\frac{x-u}{1-u}, \frac{y-u}{1-u}\right) & \text { se } x, y \epsilon[u, 1] \\ u, & \text { caso contrario }\end{cases}
$$

Para construir os neurônios unineuron e nullneuron, é necessário inicialmente propor-se uma pequena modificação na uninorma e na nullnorma, apresentada nas Equações (11) e (12).

\subsection{Unineuron}

$$
\tilde{U}(x, y ; g)= \begin{cases}g T\left(\frac{x}{g}, \frac{y}{g}\right) & \text { se } x, y \epsilon[0, g] \\ g+(1-g) S\left(\frac{x-g}{1-g}, \frac{y-g}{1-g}\right) & \text { se } x, y \epsilon[g, 1] \\ \varphi(x, y) & \text { caso contrario }\end{cases}
$$

Onde:

$$
\varphi(x, y)= \begin{cases}\max (x, y) & \text { se } g=[0,0.5) \\ \min (x, y) & \text { se } g=[0.5,1]\end{cases}
$$

O processamento do unineuron é dado pela função uni $(x, y ; g)$, onde $x \epsilon[0,1]^{n}$ e $y \epsilon[0,1]^{n}$ são definidos nas equações (15) e (16).

$$
\begin{array}{r}
u n i(x, y ; g)=\tilde{U}\left[\tilde{U}\left(x_{1}, y_{1} ;(1-g)\right),\right. \\
\left.\tilde{U}\left(x_{2}, y_{2} ;(1-g)\right), \cdots \tilde{U}\left(x_{n}, y_{n} ;(1-g)\right) ; g\right] \\
\text { uni }(x, y ; g)=\tilde{U}_{i=1}^{n}\left[\tilde{U}\left(x_{i}, y_{i} ;(1-g)\right) ; g\right]
\end{array}
$$

\section{Nullneuron}

$$
\tilde{N}(x, y ; u)= \begin{cases}T(x, y) & \text { se } u=0 \\ \frac{1}{2} & \text { se } u=\frac{1}{2} \\ S(x, y) & \text { se } u=1\end{cases}
$$

Tem-se que para $0<u<0.5$, a função $\tilde{N}$ se assemelha a uma T-norma e, para $0.5<u<1$ esta função se assemelha a uma S-norma. Assim o processamento do Nullneuron é dado pela função $\operatorname{null}(x, y ; u)$, onde $x \epsilon[0,1]^{n}$ e $y \epsilon[0,1]^{n}$ são definidos nas equações (18) e (19).

$$
\begin{array}{r}
\operatorname{null}(x, y ; u)=\tilde{N}\left[\tilde{U}\left(x_{1}, y_{1} ;(1-u)\right),\right. \\
\left.\tilde{N}\left(x_{2}, y_{2} ;(1-u)\right), \cdots \tilde{N}\left(x_{n}, y_{n} ;(1-u)\right) ; u\right] \\
\operatorname{null}(x, y ; u)=\tilde{N}_{i=1}^{n}\left[\tilde{N}\left(x_{i}, y_{i} ;(1-u)\right) ; u\right]
\end{array}
$$

As variáveis $x$ e $y$ correspondem as entradas do sistema, no presente trabalho equivale ao valor da perda e do carregamento dos alimentadores, para cada configuração, já normalizados utilizando a equação (6).
Neste trabalho os elementos identidade $g$ e $u$ foram inicializados aleatoriamente no intervalo $[0,1]$ e este valor ajustado automaticamente de acordo com a necessidade do problema. O ajuste foi realizado através da equação (20) e (21).

$$
\begin{aligned}
& g_{\text {novo }}=g_{\text {antigo }}+\Delta g \\
& u_{\text {novo }}=u_{\text {antigo }}+\Delta u
\end{aligned}
$$

Onde $\Delta g$ é a variação do elemento identidade e calculado através das equações (22) e (23).

$$
\begin{aligned}
\Delta g & =\delta \alpha_{1}[1-g]-(1-\delta) \alpha_{2} g \\
\Delta u & =\delta \alpha_{1}[1-u]-(1-\delta) \alpha_{2} u
\end{aligned}
$$

Sendo $\delta$ o sinal de reforço dado por $\delta=1-\epsilon, \epsilon$ é o erro que se deseja minimizar calculado como $\epsilon=1-y$, onde y é a saída do neurônio. $\alpha_{1}$ e $\alpha_{2}$ são a taxa de aprendizagem para os elementos identidade onde $0<\alpha_{1}<<\alpha_{2}<1$.

\section{RECONFIGURAÇÃO UTILIZANDO UNINEURON E NULLNEURON}

Para a aplicação do remanejamento de carga proposto, foi necessário primeiramente implementar, via simulação, uma rede de distribuição de energia elétrica. A rede de distribuição de 33 barras utilizada foi a rede proposta por Baran and Wu (1989). O sistema consiste em uma rede de distribuição com 1 barra de subestação e 33 barras de cargas, carga ativa total de $3.715 \mathrm{~kW}, 32$ chaves seccionadoras (normalmente fechadas) e 5 chaves de encontro (normalmente abertas). A configuração inicial apresenta perda total ativa de $243,6 \mathrm{~kW}$ e Corrente total de 0,499 p.u. O diagrama é mostrado na Figura 2:

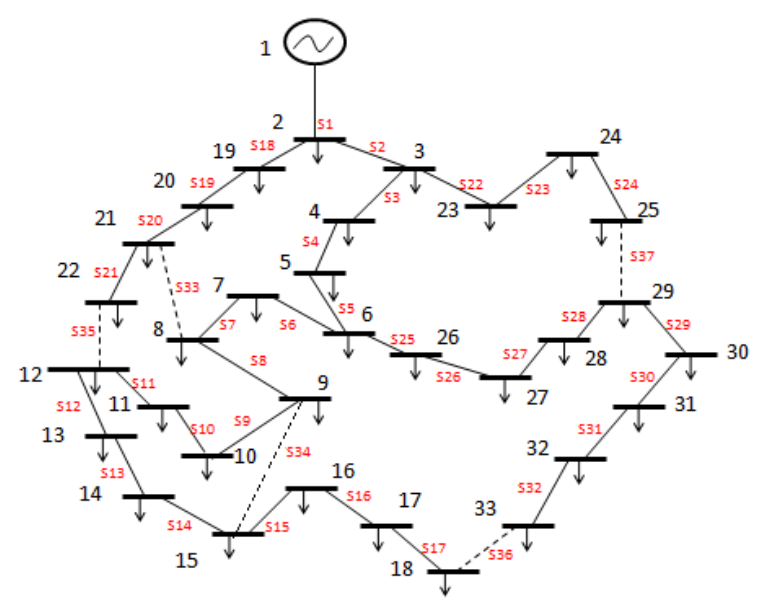

Figura 2. Sistema de 33 barras utilizado

Após a definição da rede foi possível realizar os cálculos de fluxo de potência utilizando o MATPOWER (Zimmerman et al., 2011) e aplicar o método Heurístico Branch Exchange para seleção das configurações. O resultado obtido da aplicação do método foi utilizado como entrada dos algoritmos de Bellman Zadeh, unineuron e nullneuron. O passo a passo aplicado é detalhado no fluxograma da figura 3.

O algoritmo do unineuron e nullneuron foi construído conforme detalhado no fluxograma da figura 4. A Fuzzificação dos vetores de entrada foi realizado utilizando a equação 


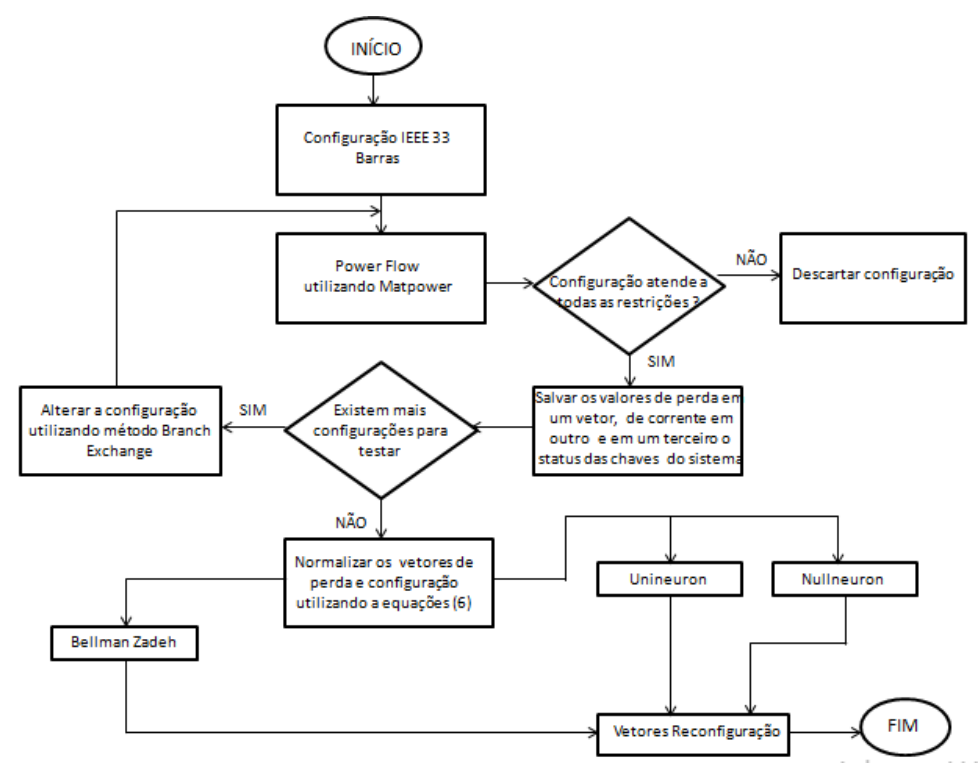

Figura 3. Metodologia adotada

(6). Os valores de $g$ e $u$ foram inicializados aleatoriamente e, como dito anteriormente, ajustados utilizando as equações (20) e (21). Os valores de $\alpha_{1}$ foi considerado igual a 0,000001 e de $\alpha_{2}$ igual a 0,00005 .

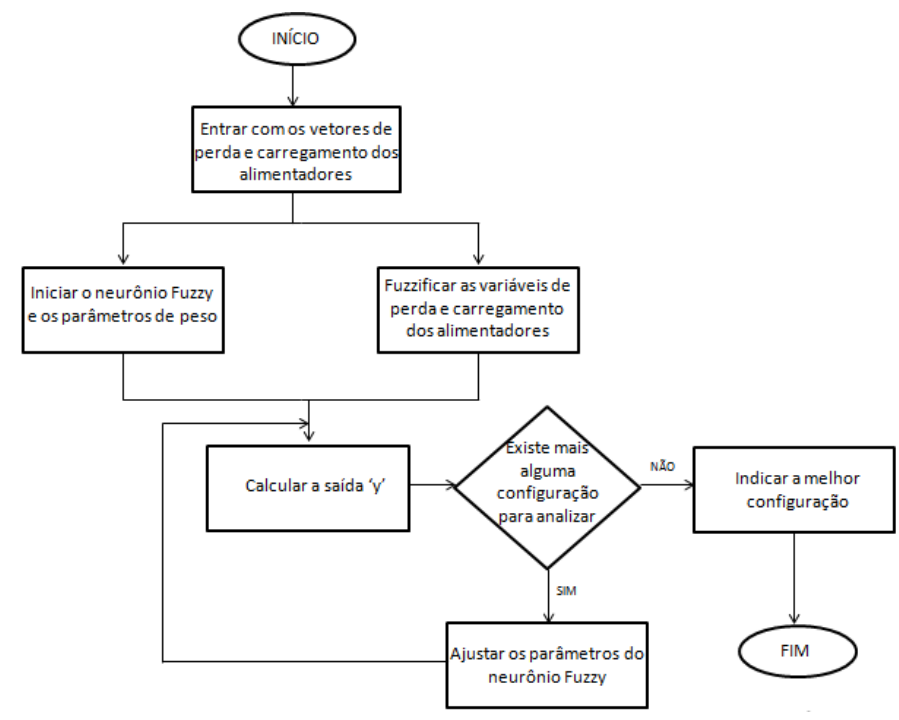

Figura 4. Metodologia unineuron e nullneuron

Os resultados utilizando os métodos propostos foram analisados. Estes resultados foram então comparados entre si e mostrados na seção seguinte.

\section{RESULTADOS}

A Tabela 2 mostra os resultados obtidos utilizando apenas o método Branch Exchange, considerando um problema monoobjetivo. A configuração que apresentou a menor perda de potência elétrica foi a de número 12 , e a de número 25 apresentou menor carregamento dos alimentadores. $\mathrm{O}$ valor de pertinência mais alto indica uma maior satisfação da solução. Sendo assim, a melhor configuração considerando apenas minimização das perdas é a 12 e a melhor considerando apenas minimização do dos alimentadores é a 25 .
A tabela 3 mostra o resultado obtido após inserir os dados nos neurônios maxmin. A redução foi calculada em relação a perda de potência elétrica da configuração inicial, que equivale a $202,68 \mathrm{~kW}$ e em relação ao carregamento inicial dos alimentadores que equivale a 0,499 p.u. Assim como esperado, foi possível perceber que o método privilegiou apenas o objetivo de minimização das perdas.

Utilizando unineuron e nullneuron, foi mostrado os resultados para diferentes pesos de cada objetivo. Os pesos utilizados foram $20 \%$ para perda e $80 \%$ para carregamento dos alimentadores, $50 \%$ para perda e $50 \%$ para carregamento dos alimentadores e $90 \%$ para perda e $10 \%$ para carregamento dos alimentadores. O resultado é mostrado na Tabela 4. Ambos os métodos apresentaram o mesmo resultado.

Como já era de se esperar, quando o carregamento dos alimentadores foi o objetivo privilegiado em uma proporção bem maior (20/80), a configuração escolhida foi a que apresentou menor carregamento dos alimentadores, a configuração 25, uma redução na perda de 7,49\% e no carregamento dos alimentadores de 38,48\%. O mesmo ocorreu quando a perda foi privilegiada em uma proporção bem maior (90/10), a configuração 12 que apresentou a maior redução de perda foi a escolhida, uma redução de $28,20 \%$ na perda e de $30,26 \%$ de carregamento dos alimentadores.

Quando ambos os objetivos foram considerados em mesma proporção (50/50), a melhor configuração foi a que ocorreu uma redução na perda de $30,94 \%$ e redução no carregamento dos alimentadores de 30,26\%. Sendo assim, ambos convergiram para a melhor topologia em que os objetivos de minimização de perda e minimização do carregamento dos alimentadores são atendidos.

A tabela 5 mostra uma comparação dos métodos utilizados neste trabalho com alguns presentes na literatura, como PSO (Particle Swarm Optimization), MPSO (Modified Particle Swarm Optimization ) e ACO (Ant Colony Optimization). Os valores de PSO, presentes na tabela, foram retirados de Dahalan and Mokhlis (2012), os de MPSO de 
Tabela 3. Melhor configuração utilizando Bellman Zadeh

Tabela 2. Resultado de cada configuração analisada utilizando Branch Exchange

\begin{tabular}{|c|c|c|c|c|c|}
\hline$n^{o}$ & $\begin{array}{c}\text { Chaves } \\
\text { abertas }\end{array}$ & $\begin{array}{c}\begin{array}{c}\text { Perda } \\
(\mathrm{kW})\end{array} \\
\end{array}$ & $\begin{array}{c}\text { Corrente } \\
\text { (p.u) }\end{array}$ & $\begin{array}{l}\text { Pert. } \\
\text { Perda }\end{array}$ & $\begin{array}{c}\text { Pert. } \\
\text { Carreg. }\end{array}$ \\
\hline 1 & $\begin{array}{c}\text { S21,S33,S34, } \\
\text { S36,S37 }\end{array}$ & 216,05 & 0,581 & 0,273 & 0,528 \\
\hline 2 & $\begin{array}{c}\text { S11,S33,S34, } \\
\text { S36,S37 }\end{array}$ & 156,79 & 0,422 & 0,472 & 0,727 \\
\hline 3 & $\begin{array}{c}\text { S10,S33,S34, } \\
\text { S36,S37 }\end{array}$ & 155,13 & 0,418 & 0,478 & 0,734 \\
\hline 4 & $\begin{array}{c}\text { S9,S33,S34, } \\
\text { S36,S37 }\end{array}$ & 153,99 & 0,384 & 0,482 & 0,799 \\
\hline 5 & $\begin{array}{c}\text { S8,S33,S34, } \\
\text { S36,S37 }\end{array}$ & 153,49 & 0,377 & 0,483 & 0,814 \\
\hline 6 & $\begin{array}{c}\text { S9,S20,S34, } \\
\text { S36,S37 }\end{array}$ & 239,33 & 0,652 & 0,195 & 0,471 \\
\hline 7 & $\begin{array}{c}\text { S7,S9,S34, } \\
\text { S36,S37 }\end{array}$ & 146,16 & 0,911 & 0,508 & 0,337 \\
\hline 8 & $\begin{array}{c}\text { S6,S9,S34, } \\
\text { S36,S37 }\end{array}$ & 145,92 & 0,38 & 0,509 & 0,808 \\
\hline 9 & $\begin{array}{c}\text { S7,S8,S9, } \\
\text { S36,S37 }\end{array}$ & 148,79 & 0,391 & 0,499 & 0,785 \\
\hline 10 & $\begin{array}{c}\text { S7,S9,S14, } \\
\text { S36,S37 }\end{array}$ & 142,17 & 0,358 & 0,522 & 0,858 \\
\hline 11 & $\begin{array}{c}\text { S7,S9,S13, } \\
\text { S36,S37 }\end{array}$ & 144,83 & 0,387 & 0,513 & 0,793 \\
\hline 12 & $\begin{array}{c}\text { S7,S9,S14, } \\
\text { S32,S37 }\end{array}$ & 139,55 & 0,364 & 0,530 & 0,843 \\
\hline 13 & $\begin{array}{l}\text { S7,S9,S14, } \\
\text { S31,S37 }\end{array}$ & 142,6 & 0,353 & 0,520 & 0,870 \\
\hline 14 & $\begin{array}{l}\text { S7,S9,S14, } \\
\text { S17,S37 }\end{array}$ & 147,54 & 0,394 & 0,503 & 0,779 \\
\hline 15 & $\begin{array}{l}\text { S7,S9,S14, } \\
\text { S24,S32 }\end{array}$ & 174,2 & 0,444 & 0,414 & 0,691 \\
\hline 16 & $\begin{array}{c}\text { S7,S9,S14, } \\
\text { S28,S32 }\end{array}$ & 139,98 & 0,348 & 0,529 & 0,882 \\
\hline 17 & $\begin{array}{c}\text { S7,S33,S34, } \\
\text { S36,S37 }\end{array}$ & 156,53 & 0,375 & 0,473 & 0,819 \\
\hline 18 & $\begin{array}{c}\text { S6,S33,S34, } \\
\text { S36,S37 }\end{array}$ & 168,2 & 0,437 & 0,434 & 0,703 \\
\hline 19 & $\begin{array}{c}\text { S7,S21,S34, } \\
\text { S36,S37 }\end{array}$ & 164,56 & 0,431 & 0,446 & 0,712 \\
\hline 20 & $\begin{array}{c}\text { S7,S8,S34, } \\
\text { S36,S37 }\end{array}$ & 147,65 & 0,376 & 0,503 & 0,816 \\
\hline 21 & $\begin{array}{l}\text { S7,S9,S34, } \\
\text { S36,S37 }\end{array}$ & 146,16 & 0,374 & 0,508 & 0,821 \\
\hline 22 & $\begin{array}{c}\text { S7,S8,S14, } \\
\text { S36,S37 }\end{array}$ & 147,29 & 0,388 & 0,504 & 0,791 \\
\hline 23 & $\begin{array}{c}\text { S7,S8,S32, } \\
\text { S34,S37 }\end{array}$ & 146,47 & 0,373 & 0,507 & 0,823 \\
\hline 24 & $\begin{array}{l}\text { S7,S8,S31, } \\
\text { S34,S37 }\end{array}$ & 154,8 & 0,308 & 0,479 & 0,997 \\
\hline 25 & $\begin{array}{c}\text { S7,S8,S30, } \\
\text { S34,S37 }\end{array}$ & 171,72 & 0,307 & 0,422 & 1,000 \\
\hline 26 & $\begin{array}{l}\text { S7,S8,S29, } \\
\text { S34,S37 }\end{array}$ & 297,15 & 1,001 & 0,000 & 0,307 \\
\hline 27 & $\begin{array}{l}\text { S7,S8,S17, } \\
\text { S34,S37 }\end{array}$ & 151,22 & 0,401 & 0,491 & 0,766 \\
\hline 28 & $\begin{array}{c}\text { S7,S8,S24, } \\
\text { S30,S34 }\end{array}$ & 193,57 & 0,378 & 0,349 & 0,812 \\
\hline 29 & $\begin{array}{c}\text { S7,S8,S28, } \\
\text { S30,S34 }\end{array}$ & 173,44 & 0,331 & 0,416 & 0,927 \\
\hline
\end{tabular}

\begin{tabular}{|c|c|c|c|c|c|}
\hline $\mathbf{n}^{\mathbf{0}}$ & $\begin{array}{c}\text { Chaves } \\
\text { abertas }\end{array}$ & $\begin{array}{c}\text { Perda } \\
(\mathbf{k W})\end{array}$ & $\begin{array}{c}\text { Corrente } \\
(\mathbf{p . u})\end{array}$ & $\begin{array}{c}\text { Red. } \\
\text { Perda } \\
\mathbf{( \% )}\end{array}$ & $\begin{array}{c}\text { Red. } \\
\text { carreg. } \\
(\mathbf{\%})\end{array}$ \\
\hline $\mathbf{1 2}$ & $\begin{array}{c}\mathbf{S 7 , S 9 , S 1 4 ,} \\
\mathbf{S 3 2 , S 3 7}\end{array}$ & 139,55 & 0,364 & 31,15 & 27,05 \\
\hline
\end{tabular}

Tabela 4. Melhores configurações utilizando utilizando unineuron e nullneuron para diferentes pesos

\begin{tabular}{|c|c|c|c|c|c|c|}
\hline $\begin{array}{c}\text { Peso } \\
\mathbf{P} / \mathbf{C} \\
(\mathbf{\%})\end{array}$ & $\mathbf{n}^{\mathbf{0}}$ & $\begin{array}{c}\text { Chaves } \\
\text { abertas }\end{array}$ & $\begin{array}{c}\text { Perda } \\
(\mathbf{k W})\end{array}$ & $\begin{array}{c}\text { Corr. } \\
(\mathbf{p . u})\end{array}$ & $\begin{array}{c}\text { Red. } \\
\text { Perda } \\
(\mathbf{\%})\end{array}$ & $\begin{array}{c}\text { Red. } \\
\mathbf{C a r r} \\
\mathbf{( \% )}\end{array}$ \\
\hline $\mathbf{2 0 / 8 0}$ & $\mathbf{2 5}$ & $\begin{array}{c}\mathbf{S 7 , S 8 , S 3 0 ,} \\
\mathbf{S 3 4 , S 3 7}\end{array}$ & 171,72 & 0,307 & 15,28 & 38,48 \\
\hline $\mathbf{5 0 / 5 0}$ & $\mathbf{1 6}$ & $\begin{array}{c}\mathbf{S 7 , S 9 , S 1 4} \\
\mathbf{S 2 8 , S 3 2}\end{array}$ & 139,98 & 0,348 & 30,94 & 30,26 \\
\hline $\mathbf{9 0 / 1 0}$ & $\mathbf{1 2}$ & $\begin{array}{c}\mathbf{S 7 , S 9 , S 1 4} \\
\mathbf{S 3 2 , S 3 7}\end{array}$ & 139,55 & 0,364 & 31,15 & 27,05 \\
\hline
\end{tabular}

Tabela 5. Comparação com métodos presentes na literatura

\begin{tabular}{|c|c|c|c|}
\hline Método & $\begin{array}{c}\text { Chaves } \\
\text { abertas }\end{array}$ & $\begin{array}{c}\text { Redução } \\
\text { Perda } \\
(\%)\end{array}$ & $\begin{array}{c}\text { Redução } \\
\text { Carreg. } \\
(\%)\end{array}$ \\
\hline $\begin{array}{c}\text { Bellman } \\
\text { Zadeh }\end{array}$ & $\mathbf{S 7 , S 9 , S 1 4 , S 3 2 , S 3 7 ~}$ & 31,15 & 27,05 \\
\hline MPSO & $\mathbf{S 7 , S 9 , S 1 4 , S 3 2 , S 3 7 ~}$ & 31,15 & 27,05 \\
\hline ACO & $\mathbf{S 7 , S 9 , S 1 4 , S 3 2 , S 3 7 ~}$ & 31,15 & 27,05 \\
\hline Unineuron & $\mathbf{S 7 , S 9 , S 1 4 , S 2 8 , S 3 2 ~}$ & 30,94 & 30,26 \\
\hline Nullneuron & $\mathbf{S 7 , S 9 , S 1 4 , S 2 8 , S 3 2 ~}$ & 30,94 & 30,26 \\
\hline PSO & $\mathbf{S 8 , S 1 7 , S 2 8 , S 3 3 , S 3 4 ~}$ & 30,92 & - \\
\hline
\end{tabular}

Atteya et al. (2017) e os do método ACO de Ghorbani et al. (2008) Ambos os métodos aqui comparados, presentes na literatura, consideraram apenas a minimização da perda e utilizaram a mesma rede do IEEE de 33 barras. Os três métodos aplicados neste trabalho apresentaram uma configuração com maior redução de perda do que a utilizando PSO. Já para os métodos MPSO e ACO a configuração escolhida foi a mesma utilizando Bellman Zadeh, o que evidência que o Método de Bellman Zadeh privilegia um único objetivo. $\mathrm{O}$ unineuron e nullneuron apresentaram menor redução de perda do que utilizando MPSO e ACO, o que é justificado pelo fato destes estarem considerando a minimização de dois objetivos em conjunto.

\section{CONCLUSÃO}

Neste trabalho foi proposto um algoritmo baseado no método unineuron e nullneuron para a solução do problema de reconfiguração de sistemas de distribuição de energia elétrica. Os resultados obtidos foram comparados com os utilizando a abordagem de Bellman Zadeh e com alguns métodos presentes na literatura.

Utilizando Bellman Zadeh, apenas uma função objetivo foi privilegiada, no caso a minimização da perda, não se mostrando um método adequado para tomada de decisão multiobjetivo. Utilizando unineuron e nullneuron os resultados mostraram soluções de boa qualidade, atendendo a todas as restrições físicas e operacionais do problema. É importante ressaltar que no contexto da tomada de decisões multiobjetivo, soluções consideradas de boa qualidade 
são aquelas que melhor representam as preferências do decisor. Ambos os métodos apresentaram também um tempo baixo de processamento computacional o que representa uma vantagem em relação aos métodos metaheurísticos.

\section{REFERÊNCIAS}

Amin Heidari, M. (2017). Optimal network reconfiguration in distribution system for loss reduction and voltage-profile improvement using hybrid algorithm of pso and aco. CIRED - Open Access Proceedings Journal, 2017(1), 2458-2461. doi:10.1049/oap-cired.2017.1007.

Atteya, I.I., Ashour, H., Fahmi, N., and Strickland, D. (2017). Radial distribution network reconfiguration for power losses reduction using a modified particle swarm optimisation. CIRED - Open Access Proceedings Journal, 2017(1), 2505-2508. doi:10.1049/oap-cired. 2017.1286.

Baran, M.E. and Wu, F.F. (1989). Network reconfiguration in distribution systems for loss reduction and load balancing. IEEE Transactions on Power Delivery, 4(2), 1401-1407. doi:10.1109/61.25627.

Bellman, R. and Zadeh, L. (1970). Decision-Making In a Fuzzy Environment. Management Science Series BApplication, 17(4), B141-B164.

Bernardon, D.P., Garcia, V.J., Ferreira, A.S.Q., Canha, L.N., and Abaide, A.R. (2009). New fuzzy multicriteria decision making algorithm to distribution network reconfiguration. In 2009 44th International Universities Power Engineering Conference (UPEC), 1-5.

Berredo, R.C., Ekel, P.Y., Martini, J.S.C., Palhares, R.M., Parreiras, R.O., and Pereira, Jr., J.G. (2011). Decision making in fuzzy environment and multicriteria power engineering problems. INTERNATIONAL JOURNAL OF ELECTRICAL POWER \& ENERGY SYSTEMS, 33(3), 623-632. doi:\{10.1016/j.ijepes.2010.12.020\}.

Chen, G., Wang, Y., Lu, G., Hu, J., You, D., Zhang, F., and He, Z. (2016). An improved load-shedding model based on power flow tracing. In 2016 12th World Congress on Intelligent Control and Automation (WCICA), 1590-1593. doi:10.1109/WCICA.2016.7578552.

Civanlar, S., Grainger, J.J., Yin, H., and Lee, S.S.H. (1988). Distribution feeder reconfiguration for loss reduction. IEEE Transactions on Power Delivery, 3(3), 1217-1223. doi:10.1109/61.193906.

Dahalan, W.M. and Mokhlis, H. (2012). Network reconfiguration for loss reduction with distributed generations using pso. In 2012 IEEE International Conference on Power and Energy (PECon), 823-828. doi:10.1109/ PECon.2012.6450331.

Ghorbani, M.A., Hosseinian, S.H., and Vahidi, B. (2008). Application of ant colony system algorithm to distribution networks reconfiguration for loss reduction. In 2008 11th International Conference on Optimization of Electrical and Electronic Equipment, 269-273. doi:10. 1109/OPTIM.2008.4602377.

Hell, M. (2008). Abordagem Neurofuzzy para Modelagem de Sistemas Dinâmicos Não Lineares. Tese de doutorado, Faculdade de Engenharia Eletrica e de Computação da Universidade Estadual de Campinas, Campinas.

Junior, J. (2014). Modelos e Metodos de Tomada de Decisões Multiobjetivo em Ambiente com Incertezas e Suas Aplicações. Tese de doutorado, Universidade Federal de Minas Gerais, Belo Horizonte.
Li, X., Zeng, Z., Zhang, Y., and Xu, X. (2007). A study of distribution load transfer operation based on gis. In 2007 International Conference on Machine Learning and Cybernetics, volume 3, 1428-1433. doi:10.1109/ ICMLC.2007.4370369.

Macedo, L. (2012). Reconfiguração de sistemas de distribuição de energia eletrica para minimização de perdas ativas totais via otimização por enxame de particulas (pso).

Merlin, A. and Back, H. (1975). Search for a minimal-loss operating spanning tree configuration in an urban power distribution system. In Proceeding 5th Power System Conference, 18.

Neto, A. and Vellasco, M. (2016). Reconfiguração de redes de distribuição utilizando restrições fuzzy. Revista de Inteligência Computacional Aplicada, 10.

Oliveira, C. (2016). Algoritmo para reconfiguração de sistema de distribuição de energia eletrica baseado em evolução diferencial.

Pandiarajan, K. and Babulal, C.K. (2011). Overload alleviation in electric power system using fuzzy logic. In 2011 International Conference on Computer, Communication and Electrical Technology (ICCCET), 417-423. doi:10.1109/ICCCET.2011.5762511.

Pedrycz, W. and Rocha, A.F. (1993). Fuzzy-set based models of neurons and knowledge-based networks. IEEE Transactions on Fuzzy Systems, 1(4), 254-266. doi:10. 1109/91.251926.

Peponis, G.J. and Papadopoulos, M.P. (1997). New dynamic, branch exchange method for optimal distribution system planning. IEE Proceedings - Generation, Transmission and Distribution, 144(3), 333-339. doi: 10.1049/ip-gtd:19971135.

Romano, R., Gaspar, L., Guedes, L., Lisboa, A., and Vieira, D. (2013). Ferramenta multicriterio para reconfiguração de redes radiais de distribuição de energia eletrica. In Congresso de Inovação Tecnologica em Energia Eletrica (VII CITENEL).

Silva, F. (2010). Reconfiguração otima de sistemas de distribuição de energia eletrica baseado no comportamento de colônia de formigas. Tese de doutorado, Escola de Engenharia de São Carlos de São Paulo, São Carlos.

Tofis, Y., Timotheou, S., and Kyriakides, E. (2017). Minimal load shedding using the swing equation. IEEE Transactions on Power Systems, 32(3), 2466-2467. doi: 10.1109/TPWRS.2016.2614886.

Yager, R. (2001). Uninorms in fuzzy systems modeling. FUZZY SETS AND SYSTEMS, 122(1), 167-175. doi: $\{10.1016 / \mathrm{S} 0165-0114(00) 00027-0\}$.

Yager, R. and Rybalov, A. (1996). Uninorm aggregation operators. FUZZY SETS AND SYSTEMS, 80(1), 111120. doi: $\{10.1016 / 0165-0114(95) 00133-6\}$.

Yan, J., Li, C., and Liu, Y. (2017). Adaptive load shedding method based on power imbalance estimated by ann. In TENCON 2017 - 2017 IEEE Region 10 Conference, 2996-2999. doi:10.1109/TENCON.2017.8228375.

Zimmerman, R.D., Edmundo Murillo-Sanchez, C., and Thomas, R.J. (2011). MATPOWER: Steady-State Operations, Planning, and Analysis Tools for Power Systems Research and Education. IEEE TRANSACTIONS ON POWER SYSTEMS, 26(1), 12-19. doi:\{10.1109/ TPWRS.2010.2051168\}. 